Classification

Physics Abstracts

$46.30 \mathrm{~L}-03.40 \mathrm{D}-47.20$

\title{
Role of boundary conditions on mode selection in a buckling instability
}

\author{
M. Boucif, J. E. Wesfreid and E. Guyon \\ Laboratoire d'Hydrodynamique et de Mécanique Physique (*), \\ Ecole Supérieure de Physique et Chimie Industrielle de Paris, 10, rue Vauquelin, \\ 75231 Paris Cedex 05, France
}

(Reçu le 2 février 1984, accepté le 7 mars 1984)

Résumé. - En variant les conditions aux bords chargés, on sélectionne de façon différente les modes instables accessibles. La sélection de modes est reliée à des analyses non linéaires de ces problèmes. Une comparaison est établie avec la sélection des modes dans des instabilités convectives.

\begin{abstract}
We present an experimental study on the selection of the wave number in the buckling of a thin elastic rectangular plate, subjected to a compressive force while being held laterally. Boundary conditions act selectively through non-linear mechanisms to restrict the accessible states above threshold.
\end{abstract}

We report experiments on mode selection in the mechanical buckling instability of a thin elastic plate subjected to uniaxial compression.

The geometry of this experiment is sketched on figure 1 . The lateral edges of the elastic plate are prevented from deflecting perpendicularly to the plane of the plate, but are free to stretch or extend. We assume no bending moments and shear along the edges. An axial load $F$ is applied along $x$.

In this case, if there is no initial deflection - geometrical imperfection [1] - in the unloaded state, the plate remains flat when the load increases from zero. The relation between the uniform stress ( $\sigma=F / h e$, where $h$ is the width and $e$ is the thickness of the plate) and the axial strain (relative shortening) is given by Hooke's linear law.

Above a certain loading - or critical force $F_{\mathrm{c}}$ - buckling occurs continuously and reversibly and out-of-plane deflections develop with spatially periodic structures of alternate humps and wells of length approximately equal to $h$. In the buckled state the axial strain is increased with respect to the unbuckled state; consequently the effective stiffness (inverse of the slope in the shortening-load curve) is diminished.

We are interested in the selection mechanism of these periodic modes. Changes of the waveform was initially observed by Stein [2] when the loaded side was clamped, for rectangular-plates of longitudinal extent $L$ and aspect ratio $\Gamma=L / h=5.7$; he observed a jump from 5 to 6 modes, by

(*) ERA 1000 du CNRS. 


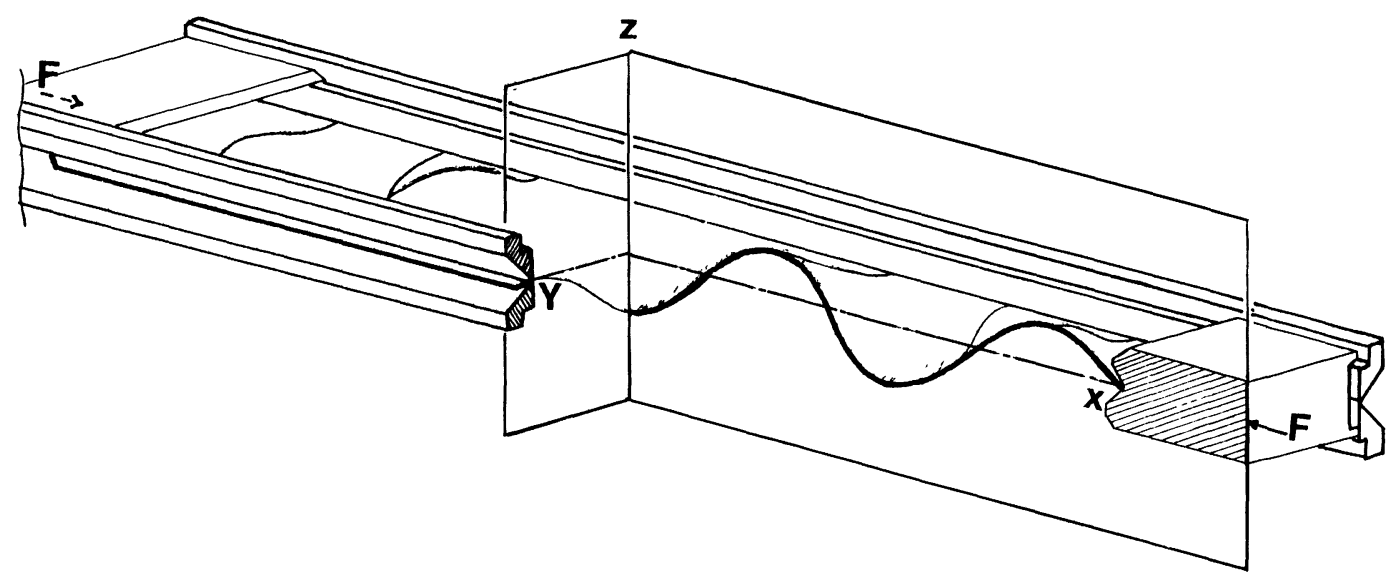

Fig. 1. - Experimental. The plate is held laterally along parallel lines $|y|=h / 2$ and subjected to an uniaxial compressive force $F$ along the $x$ axis.

increasing the load. This phenomenon, sometimes called secondary buckling [3], was reexamined by Pomeau [4], who related this jump to the non-linear influence of boundary conditions on the selection of modes among the accessible ones deduced from a linear stability analysis. Our experiment gives a study of this multiplicity of deformation modes for different boundary conditions and extends a preliminary report [5].

The geometry of the experiment is shown in figure 1. The dimensions of the brass alloy (" crisocal ») plate used are : length $L=180 \mathrm{~mm}$, width $h=20 \mathrm{~mm}$, thickness $e=0.1 \mathrm{~mm}$. The plate is guided along its unloaded longitudinal sides with boundary conditions

$$
w=\frac{\partial^{2} w}{\partial y^{2}}=0 \text { for }|y|=h / 2,|x|<L / 2
$$

$w(x, y)$ is the deflection of the plate. The deformation is measured with a mechanical gauge, of inductive type and can also be observed by direct optical visualization and by a Moiré reflection technique.

The plate is subjected to a constant compressive force, $F$, along its short sides. The two end boundary conditions, used in the present study, sketched on figures $2 \mathrm{a}$ and $2 \mathrm{~b}$, are, for $|x|=L / 2$ and $|y| \leqslant h / 2$ :

(a) simply supported (S.S.) $: w=\frac{\partial^{2} w}{\partial x^{2}}=0 ; \frac{\partial w}{\partial x} \neq 0$

(b) clamped case (C.) $: w=\frac{\partial w}{\partial x}=0 ; \frac{\partial^{2} w}{\partial x^{2}} \neq 0$.

They can be expressed as limits of general boundary conditions,

$$
w=0 ; \quad \frac{\partial w}{\partial x}+k \frac{\partial^{2} w}{\partial x^{2}}=0
$$

where the length " $k$ » is a measure of the efficiency of the clamping at both ends : $k=\infty, 0$ for cases $a, b$.

In this letter we present the first results concerning the stability of deformation modes in the S.S. case $(a)$. This boundary condition applied to the loaded sides is quite difficult to obtain exactly experimentally. In addition the inhomogeneity of the pressure distribution along the short side 
reduces the " effective width " [1] of the plate above the buckling threshold and consequently reduces the ultimate load on the plate. By applying a layer of epoxy resin on the flanges near the edges, we have been able to reduce this effect up to larger pressures $F \sim 3$ times the critical force $F_{\mathrm{c}}$.

We consider the solution of the Füppl-Von Karman equation [6] for the linear instability problem, above $F_{c}$. With the S.S. boundary conditions (2a) considered here, a sine-wave solution is expected to apply (Fig. 2a) :

$$
w(x, y)=w_{0} \sin (q x / h) \cdot \sin (\pi y / h) .
$$

The boundary conditions for the stress distribution [4] do not influence the resolution of the linear problem, when there are not restrictions to the lateral expansion of the plate.

The lowest threshold of linear instability is given by [6] :

$$
\tilde{F}_{\mathrm{c}}=4 \pi^{2} ; \quad \tilde{q}_{\mathrm{c}}=\pi
$$

where the force $F$ and the wave number of distortion, $q$, have been expressed in dimensionless form

$$
\tilde{F}=12\left(1-v^{2}\right) h F / E e^{3} ; \quad \tilde{q}=q h
$$

$E\left(=13000 \mathrm{daN} / \mathrm{mm}^{2}\right)$ and $v(=0.34)$ are the Young and Poisson moduli of the brass plate.

In the S.S. experiments, we measure a value

$$
F_{\mathrm{c}}=25.5 \pm 1.8 \mathrm{~N} \quad \text { or } \quad \tilde{F}_{\mathrm{c}}=41.6 \pm 2.9
$$

not far from the theoretical threshold given by (4).

The solution of the linear problem as a function of the wave number $q$ is given by the following equation, where $F$ and $q$ are given in dimensionless form as in the rest of the paper :

$$
F_{\mathrm{c}}(q) / F_{\mathrm{c}}\left(q_{\mathrm{c}}\right)=\left(q^{2}+\pi^{2}\right)^{2} / 4 \pi^{2} q^{2}
$$

and is the marginal stability curve $(\mathrm{L})$ in the diagram of figure 3 . We have plotted a branch of

(2a)

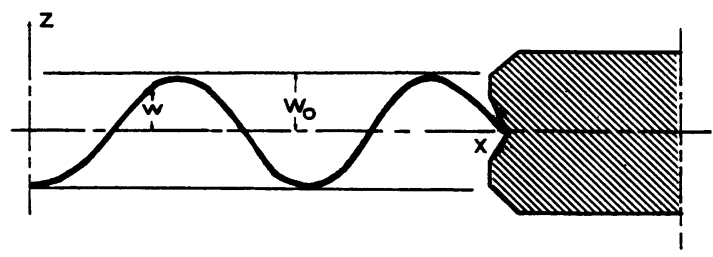

(2b)

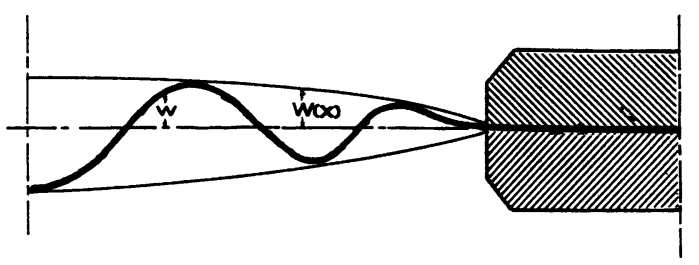

Fig. 2. - Gives schematically the two boundary conditions used : (2a) simply supported : the deformation above threshold is sinusoidal with a constant amplitude $w_{0} ;(2 \mathrm{~b})$ clamped : the shape of the deformation just above threshold which satisfies the boundary conditions $(2 b)$ has a modulated envelope. 


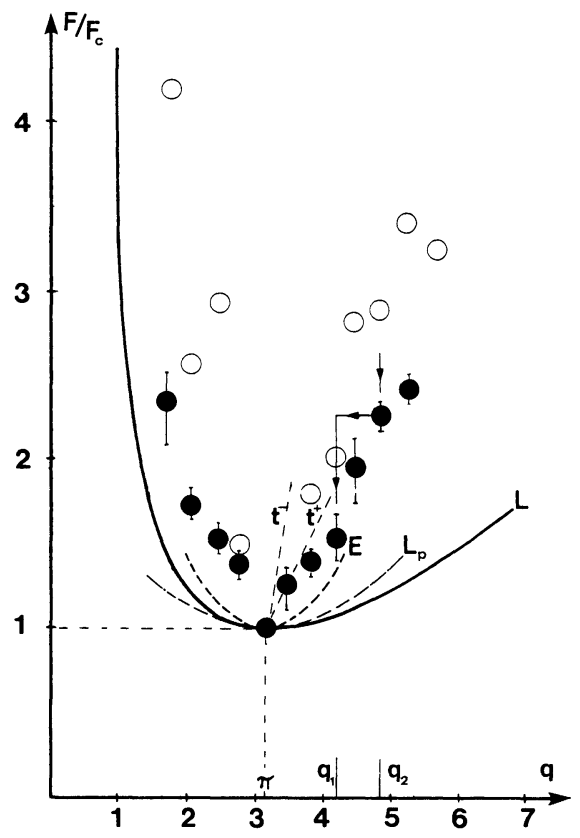

Fig. 3. - Stability diagram giving the force versus wave vector of the distortion, in units $F / F_{\mathrm{c}}$. The points above the curve $\mathrm{L}$ are linearly unstable. The full dots are the experimental data giving the lower limit of non-linear stability with boundary conditions (2a). They lie below the open dots data given in Ref. [5] which were obtained in a similar fashion for the boundary conditions (2b). (Note the factor of 2 to 3 difference along the vertical direction.) The theoretical lines $\left(\mathrm{t}^{+}, \mathrm{t}^{-}\right)$were obtained in this last case, in Ref. [4]. The sequence indicated by 3 arrows and leading to a decrease of $q$ from $q_{1}$ to $q_{2}$ as the applied force is progressively reduced corresponds to boundary conditions (2a). The parabolic curve $E$ gives the lower limit of stability defined by Eckhaus criterion near $F_{\mathrm{c}}$. Its range of validity is expected to correspond to that of the parabola $\mathrm{L}_{\mathbf{p}}$ of linear stability.

parabola $\left(\mathrm{L}_{\mathrm{p}}\right)$ next $\left(F_{\mathrm{c}}, q_{\mathrm{c}}\right)$. It corresponds to the validity of a « parabolic » approximation using expansions in $\left(F-F_{\mathrm{c}}\right),\left(q-q_{\mathrm{c}}\right)$

$$
\varepsilon_{\mathrm{c}}(q)=\left[F_{\mathrm{c}}(q)-F_{\mathrm{c}}\left(q_{\mathrm{c}}\right)\right] / F_{\mathrm{c}}\left(q_{\mathrm{c}}\right) \simeq \xi_{0}^{2}\left(q-q_{\mathrm{c}}\right)^{2}
$$

with $\xi_{0}^{2}=1 / \pi^{2}$.

The experimental procedure used to obtain the stable non-linear deformation modes is as follows : we apply a force sufficiently larger than $F_{\mathrm{c}}$. A given wave number $q_{1}$ (see Fig. 3) is « forced» by applying temporarily a gentle pressure perpendicular to the plate, to the points corresponding to the maxima of amplitude. Then the force is slowly decreased. At a lower value, still larger than $F_{\mathrm{c}}\left(q_{1}\right)$, a jump in the solution is obtained, which corresponds to the suppression of one mode of the structure and to a discontinuous transition to a state of lower wave number $q_{2}$, if the initial value $q_{1}$ is larger than $q_{\mathrm{c}}$. In such a case, we " hear » a characteristic « click » due to the mechanical process involved in the resorption of two of bulges.

If $q_{1}$ is smaller than $q_{\mathrm{c}}$, a transition leading to a larger value $q_{2}$ takes place when the force is small enough. It involves the nucleation of an additional mode, occurring initially as a dip on the top of a bulge as may be observed with the deflection gauge. This non-linear limit of stability is defined by a set of black dots on the diagram of figure 3 , strictly above the linear limit (L). This behaviour is reminiscent of the Eckhaus instability mechanism [7] of hydrodynamic flows which is a 
non-linear stability mechanism, caused by a spontaneous amplification of perturbations parallel to the periodic structure (phase perturbations) of compression and expansion of the periodic modes.

In the lowest order non-linear approximation, the Eckhaus limit can be written, as in other instabilities with spatial organization, by the parabolic approximation

$$
\varepsilon_{\mathrm{E}}(q)=3 \xi_{0}^{2}\left(q-q_{\mathrm{c}}\right)^{2} .
$$

For comparison, this limit is indicated on the figure 3 by the dashed line $\mathrm{E}$; it is a parabola with a curvature larger by $\sqrt{3}$ than the linear one given by equation (7). This approximation applies for $\varepsilon=F-F_{\mathrm{c}} / F_{\mathrm{c}} \ll 1$, so it is not valid for our experimental points which are further above $F_{\mathrm{c}}$.

As far as we know, explicit calculations in the full elastic post buckling region (or strongly non-linear case) which could be compared with our experimental points do not exist yet.

The solution for the clamped case is expected to be different from the previous one : in this case, the sine-wave (3) with constant amplitude does not satisfy the boundary conditions (2b). In the immediate vicinity of the threshold, the envelope of the modulation has a shape sketched on figure $2 \mathrm{~b}$ [8]. This amplitude modulation is responsible for the shift in the value of the critical force $F_{\mathrm{c}}$. The influence of the " boundary layer " at the clamped sides may be easily understood from a Landau-Ginzburg expansion with a gradient term, applied to this instability. It leads to the result [8]: $F_{\mathrm{c}}(\Gamma) \simeq F_{\mathrm{c}}(\infty)\left[1+\pi^{2} \xi_{0}^{2} / \Gamma^{2}\right]$; in our case, in dimensionless units $F_{\mathrm{c}}(\Gamma)=$ $4 \pi^{2}\left[1+1 / \Gamma^{2}\right]$ gives an approximately analytical function, in agreement with numerical calculations [9].

Using the same procedure as for a S.S. plate, we have obtained experimentally in reference [5] the non-linear limit of stability in this case. The results are indicated in figure 3 by open dots above the full dots obtained for the S.S. case. Note the large vertical difference between data obtained for the same wave-numbers in the two configurations. The role of clamped sides in the boundary conditions ( $2 \mathrm{~b}$ ) with modulated amplitude, was shown [4] to lead a band of the possible modes of deformation restricted, near the instability minimum, to a cone angle : limit straight lines $\mathrm{t}^{+}$and $\mathrm{t}^{-}$on figure 3 . We note on the figure that the experimental results do not agree quantitatively with the theory. In reference [5] we had suggested the possible role of imperfections in the experimental boundary conditions. Recently Potier-Ferry [10] has extended the theoretical analysis of the problem to the buckling of a beam with non-ideal clamping using the general boundary conditions (2c). He has found that the theoretical limit $\mathrm{t}^{-}$changes slope when the clamping parameter $k$ increases, in the direction of the experimental data (points to the left of $q_{\mathrm{c}}$ ) in qualitative agreement with our experimental findings.

In conclusion, the comparison between both sets of experiments has shown :

i) a broader range of non-linear modes in the simply supported case than in the clamped one ;

ii) a different mechanism for the jump between solutions in the two cases : for (S.S.) conditions (2a), it takes place in the central region of the plate by an addition or subtraction of one mode (two bulges) of the structure. In the $(C$.) case $(2 b)$, it occurs at the ends of the plates where the deformation is only of second order in $x$ and corresponds to a variation by only one bulge (half a wave length) at a time.

There is a striking correspondence between this problem and the Rayleigh-Bénard problem of convection of fluids heated from above which is the typical example of hydrodynamic instabilities with periodic spatial structure. The selection of wavenumber in this instability has brought about much controversy [11], until it was shown that boundary conditions played a crucial role in the selection of modes among those accessible from linear stability criteria [4, 12].

The present experiment in elastic buckling (potential system) provides a simple illustration of the influence of boundary conditions on mode selection. It offers the possibility to vary these conditions, and has the advantage to handle with strictly two-dimensional structures, without transverse destabilizing mechanisms which are found to dominate in the Rayleigh-Bénard example. 
However the role of imperfections or non-homogeneous boundary conditions is very sensitive in mechanical experiments and further technical progress must be accomplished before an experiment, approaching the " critical » domain, can be made.

Let us also note that some recent works [13] have proposed generalization of the boundary conditions $(2 \mathrm{c})$ in the weakly non-linear region. Their results give qualitative indications for deviations from the Eckhaus limit in the direction of our experiments in the (S.S.) case.

\section{Acknowledgments.}

We thank J. C. Charmet, M. Clement, Y. Pomeau, M. Potier-Ferry and S. Zaleski for help and discussions on this project.

This work has been done with help from « Research award Jean Langlois ».

\section{References}

[1] KoIter, W. T., Technical report AFFDL-TR, (1970) 70-25.

[2] Stein, M., NACA Tech. Rep., (1959) R-40.

[3] Nakamura, T. and Uetani, K., Int. J. Mech. Sci. 21 (1979) 265, and references herein.

[4] Pomeau, Y., J. Physique Lett. 42 (1981) L-1.

[5] Clément, M., Wesfreid, J. E., Guyon, E., C. R. Acad. Sci. Paris II 293 (1981) 87.

[6] Landau, L. and Lifchitz, L., Theory of Elasticity, § 14 (Pergamon Press, N. Y.) 1964.

BRUSCH, D. O. and AlmoRTH, B. O., Buckling of bars, plates and shells (McGraw Hill-Kigakusha) 1975.

[7] EckHAus, W., Studies in non-linear stability theory (Springer Verlag) 1965.

[8] A similar type of modulation was obtained for the Rayleigh-Bénard thermal convection of fluids with lateral side walls.

Wesfreid, J. E., Pomeau, Y., Dubois, M., Normand, C., Bergé, P., J. Physique 39 (1978) 725.

[9] GÉRARD, G. and BECKER, H., NACA Technical note 3781 (1957).

[10] Potier-Ferry, M., in Collapse : the buckling of structures in theory and practice, ed. by J. M. T. Thompson and G. W. Hunt (Cambridge University Press) 1983, p. 149.

[11] Koschmieder, E. L., in Convective transport and instability phenomena, ed. by J. Zierep et $\mathbf{H}$. Oertel (G. Braun-Karlsruhe) 1982, p. 39.

[12] Pomeau, Y. and Zaleski, S., J. Physique 42 (1981) 515.

ZALESKI, S., Thèse de 3e cycle, Université Paris VI (1980).

Cross, M. C., Daniels, P. G., Hohenberg, P. C., Siggia, E. C., J. Fluid Mech. 127 (1983) 155.

[13] Kramer, L. and Hohenberg, P. C. and Zaleski, S., in Cellular structures in the instabilities, ed. by J. E. Wesfreid and S. Zaleski (Springer Verlag) 1984. 